\title{
A IMPORTÂNCIA DO MÉTODO DE HARTREE NO ENSINO DE QUÍMICA QUÂNTICA
}

\author{
Silmar A. do Monte* e Elizete Ventura
}

Departamento de Química, Universidade Federal da Paraíba, 58036-300 João Pessoa - PB, Brasil

Recebido em 10/5/10; aceito em 28/9/10; publicado na web em 9/2/11

\begin{abstract}
THE IMPORTANCE OF HARTREE'S METHOD IN TEACHING QUANTUM CHEMISTRY. Hartree's original ideas are described. Its connection with electrostatics can be explored in order to decrease the gap between teaching of Physics and Chemistry. As a consequence of its simplicity and connection with electrostatics, it is suggested that Hartree's method should be presented before the Hartree-Fock method. Besides, since the fundamental concepts of indistinguishibility of electrons along with the antissimetry of the wave function are missing in the Hartree's product, the method itself can be used to introduce these concepts. Despite the fact that these features are not included in the trial wavefunction, important qualitatively correct results can be obtained.
\end{abstract}

Keywords: Hartree's method; teaching quantum chemistry.

\section{INTRODUÇÃO}

O método de Hartree 12 constitui o primeiro método a utilizar um procedimento numérico iterativo para cálculos de estrutura eletrônica. Podemos afirmar que este método representa o 'pontapé' inicial dos principais métodos de estrutura eletrônica ditos ab initio, uma vez que foi o precursor do método de Hartree-Fock, como explicaremos mais adiante. Embora a sua aplicação tenha se restringido a átomos, as principais ideias contidas nele podem ser de grande importância para o ensino de Química Quântica.

A nosso ver não tem sido dada a devida importância a este método. O principal motivo (o que é completamente justificável) parece ser o fato de não incorporar, na sua formulação, os princípios da indistinguibilidade das partículas quânticas (no caso em questão, elétrons) e o da antissimetria da função de onda eletrônica. É claro que não podemos deixar de ressaltar as limitações inerentes ao método, mas também não podemos desprezar o seu valor. Além disso, as próprias limitações do método representam uma ótima oportunidade para explicar aos estudantes a importância destes dois conceitos fundamentais em Química Quântica.

De acordo com o que mostraremos neste artigo, o valor didático do método de Hartree reside, a nosso ver, em quatro principais aspectos: (1) pode ser apresentado como simplesmente mais um caso do problema de uma partícula se movendo sob a ação de uma força central (ref. 3, seção 6.1), problema este que é fundamental na resolução de Equação de Schrödinger para os átomos hidrogenoides; (2) pode ser conectado a alguns resultados simples da eletrostática, o que pode ajudar a aproximar o ensino de Física e o de Química; (3) o significado físico do conceito de orbitais atômicos, no âmbito de um ambiente multieletrônico; (4) a quebra da degenerescência dos orbitais atômicos de diferentes números quânticos $l$, como resultado da repulsão intereletrônica. Este resultado também leva ao conceito de energia de uma configuração.

\section{O PROBLEMA DE UMA PARTÍCULA SOB A AÇÃO DE UMA FORÇA CENTRAL (ref. 3, seção 6.1)}

Uma força central é aquela derivada de uma função de energia potencial esfericamente simétrica, ou seja, de um potencial do tipo $\mathrm{V}=\mathrm{V}(\mathrm{r})$. Este tipo de potencial leva à seguinte expressão para $\mathrm{a}$ força central.

$$
\vec{F}=-\frac{d V(r)}{d r} \frac{\vec{r}}{r}
$$

Como podemos observar a partir desta equação a força é dirigida na direção radial, no sentido oposto ao do raio vetor.

O operador Hamiltoniano da partícula de massa $m$ é dado por:

$$
\hat{H}=\widehat{T}+\hat{V}=-\left(\hbar^{2} / 2 m\right) \nabla^{2}+V(r)
$$

em que $\nabla^{2}=\partial^{2} / \partial \mathrm{x}^{2}+\partial^{2} / \partial \mathrm{y}^{2}+\partial^{2} / \partial \mathrm{z}^{2}$ é o operador Laplaciano em coordenadas cartesianas.

Neste ponto é importante ressaltar para o aluno a importância de se trabalhar no sistema adequado de coordenadas. As autofunções e os autovalores só podem ser obtidos se utilizarmos coordenadas esféricas. Este sistema de coordenadas é sugestivo, uma vez que o sistema tem simetria esférica. Com isso o Laplaciano se torna (ref.4, p. 367):

$$
\nabla^{2}=\frac{\partial^{2}}{\partial r^{2}}+\frac{2}{r} \frac{\partial}{\partial r}+\frac{1}{r^{2}} \frac{\partial}{\partial \theta^{2}}+\frac{1}{r^{2}} \cot g \theta \frac{\partial}{\partial \theta}+\frac{1}{r^{2} \operatorname{sen}^{2} \theta} \frac{\partial^{2}}{\partial \phi^{2}}
$$

O capítulo 5 do livro do Levine ${ }^{3}$ mostra a expressão do operador quadrado do momento angular $\left(\hat{L}^{2}\right)$ em coordenadas esféricas

$$
\hat{L}^{2}=-\hbar^{2}\left(\frac{\partial}{\partial \theta^{2}}+\cot g \theta \frac{\partial}{\partial \theta}+\frac{1}{\operatorname{sen}^{2} \theta} \frac{\partial^{2}}{\partial \phi^{2}}\right)
$$

Outro ponto importante que merece a atenção do aluno é o de que a transformação de coordenadas $(\mathrm{x}, \mathrm{y}, \mathrm{z})$ para $(\mathrm{r}, \theta, \phi)$ faz com que o operador $\hat{L}^{2}$ dependa apenas de duas coordenadas, $\theta$ e $\phi$, e não mais de três (x, y e z).

De posse destes dois resultados podemos modificar a expressão do operador Laplaciano em termos do momento angular

$$
\nabla^{2}=\frac{\partial}{\partial r^{2}}+\frac{2}{r} \frac{\partial}{\partial r}-\frac{1}{r^{2} \hbar^{2}} \hat{L}^{2}
$$


Consequentemente, também podemos expressar o operador Hamiltoniano em termos do operador momento angular:

$$
\hat{H}=-\frac{\hbar^{2}}{2 m}\left(\frac{\partial}{\partial r^{2}}+\frac{2}{r} \frac{\partial}{\partial r}\right)+\frac{1}{2 m r^{2}} \hat{L}^{2}+V(r)
$$

Esta forma de expressar o Hamiltoniano é bastante conveniente, pois, de posse das autofunções de $\hat{\mathrm{L}}^{2}$ (os harmônicos esféricos), é bem mais fácil para o aluno perceber como chegar à separação da Equação de Schrödinger em duas equações, uma para a parte angular e outra para a parte radial.

Também está mostrado de forma bastante clara na seção 6.3 do livro do Levine ${ }^{3}$ que um problema de duas partículas (como, por exemplo, o de átomos hidrogenoides) sempre pode ser separado em dois problemas de uma partícula, em que uma corresponde à partícula livre com massa igual à massa total do sistema $(M)$, e a outra é uma partícula (fictícia) de massa igual à massa reduzida do sistema $(\mu)$,movendo-se sob a ação de uma força central, cuja intensidade só depende da distância entre as partículas (Equação 1). Neste ponto, é importante lembrar ao aluno o que esta separação significa, ou seja, lembrar que o operador Hamiltoniano do sistema de duas partículas pode ser escrito como a soma dos Hamiltonianos das duas partículas mencionadas anteriormente. Também é importante lembrar que esta forma de escrever o Hamiltoniano leva naturalmente a uma função de onda na forma de um produto entre as funções de onda das duas partículas, ou seja,

$$
\Psi\left(x_{1}, y_{1}, z_{1}, x_{2}, y_{2}, z_{2}\right)=\Psi\left(X_{c m}, Y_{c m}, Z_{c m}\right) \Psi(x, y, z)
$$

em que as coordenadas na primeira função são as coordenadas do centro de massa, ao passo que as coordenadas na segunda função (que corresponde à função de onda da partícula de massa $\mu$ ) são as coordenadas relativas entre as duas partículas. Daí segue que o Hamiltoniano referente ao movimento relativo corresponde àquele dado na Equação 6, com $m$ substituído por $\mu$.

As autofunções simultâneas de $\hat{L}^{2}$ e $\hat{L}_{\mathrm{z}}$ são chamadas de harmônicos esféricos, e satisfazem às Equações 8 e 9 :

$$
\begin{gathered}
\hat{L}^{2} Y_{\ell, m_{\ell}}(\theta, \phi)=\ell(\ell+1) \hbar^{2} Y_{\ell, m_{\ell}}(\theta, \phi) \\
\hat{L}_{z} Y_{\ell, m_{\ell}}(\theta, \phi)=m_{\ell} \hbar Y_{\ell, m_{\ell}}(\theta, \phi)
\end{gathered}
$$

em que $l=0,1, \ldots$ e $m_{l}=-l,-l+1, \ldots, 0,1, \ldots, l-1, l$ Os harmônicos esféricos (ortonormalizados) são dados por (ref. 5, p. 103):

$Y_{\ell, m_{\ell}}(\theta, \phi)=\frac{(-1)^{\ell}}{2^{\ell} \ell !}\left[\frac{(2 \ell+1)\left(\ell-\left|m_{\ell}\right|\right) !}{4 \pi\left(\ell+\left|m_{\ell}\right|\right) !}\right]^{1 / 2}\left(1-y^{2}\right)^{\left|m_{\ell}\right| 2} \frac{d^{\ell+\left|m_{\ell}\right|}}{d y^{\ell+\left|m_{\ell}\right|}}\left(1-y^{2}\right)^{\ell} e^{i m_{\ell} \phi}(10)$

em que $y=\cos \theta$ e $\sqrt{1-y^{2}}=\operatorname{sen} \theta$. O nome dado a este conjunto de funções (bem antes do advento da Mecânica Quântica) deve-se ao fato de as mesmas também corresponderem aos modos normais de vibração de uma esfera elástica (ref. 5, p. 103).

Apresentado tudo isto, o trabalho que resta é encontrar as funções de onda que satisfazem à seguinte Equação de Schrödinger independente do tempo:

$$
\hat{H} \Psi(r, \theta, \phi)=E \Psi(r, \theta, \phi)
$$

em que $\Psi(r, \theta, \phi)$ corresponde à segunda função de onda dada na Equação 7 (ou seja, a função de onda referente ao movimento relativo do sistema), porém expressa em coordenadas esféricas, e H é o operador Hamiltoniano dado na Equação 6.

Uma vez que os operadores $L^{2}$ e $L_{\mathrm{z}}$ não envolvem a coordenada $r$, podemos multiplicar os harmônicos esféricos por uma função qualquer de $r, R(r)$, e ainda assim a função resultante continuar sendo autofunção de $\hat{L}^{2}$ e $\hat{L}_{\mathrm{z}}$. A forma do Hamiltoniano dada na Equação 6 é muito conveniente para se chegar a uma forma geral para a solução da Equação 11. Se assumirmos que $\Psi(r, \theta, \phi)$ é da forma $\Psi(r, \theta, \phi)=\mathrm{R}(\mathrm{r}) Y_{l, m_{l}}(\theta, \phi)$ e substituirmos na Equação 11 poderemos eliminar a parte angular:

$$
\hat{H} \Psi=-\frac{\hbar^{2}}{2 \mu}\left(R^{\prime \prime}+\frac{2}{r} R^{\prime}\right) Y_{\ell, m_{\ell}}+\frac{\ell(\ell+1) \hbar^{2}}{2 \mu r^{2}} R Y_{\ell, m_{\ell}}+V(r) R Y_{\ell, m_{\ell}}=E R Y_{\ell, m_{\ell}}
$$

Como $Y_{l, m_{l}} \neq 0$ podemos dividir os dois lados da equação por $Y_{l, m l}$, obtendo:

$$
-\frac{\hbar^{2}}{2 \mu}\left(R^{\prime \prime}+\frac{2}{r} R^{\prime}\right)+\frac{\ell(\ell+1) \hbar^{2}}{2 \mu r^{2}} R+V(r) R=E R
$$

Esta é a equação que a função $R(r)$ deve satisfazer para obtermos a função de onda completa, referente ao problema de uma partícula de massa $\mu$ se movendo sob a ação de uma força central, problema este em que os átomos hidrogenoides se encaixam, após efetuarmos a separação do movimento do centro de massa. A conclusão importantíssima a que podemos chegar é que, para qualquer problema de uma partícula sob a ação de uma força central, as soluções sempre podem ser escritas na forma $\Psi(r, \theta, \phi)=\mathrm{R}(\mathrm{r}) Y_{l, m l}(\theta, \phi)$, em que a função $R(r)$ satisfaz à Equação 12. Sendo assim, uma vez conhecido $V(r)$ podemos, em princípio, obter $R(r)$. O termo força central muitas das vezes é substituído por campo central.

No caso dos átomos hidrogenoides $V(r)$ é simplesmente o potencial Coulômbico atrativo entre o elétron e o núcleo, ou seja, $V(r)=-\frac{Z e^{2} k}{r}$, com $k=\frac{1}{4 \pi \varepsilon_{0}}$. Neste caso específico os autovalores (energias) da Equação 11 dependem apenas do número quântico principal $n$, e coincidem com os níveis de energia de Bohr. O fator radial é dado por (ref. 3, p. 144):

$$
R_{n \ell}(r)=r^{\ell} e^{-Z r / n a} \sum_{j=0}^{n-\ell-1} b_{\ell} r^{\ell}
$$

em que $\mathrm{a}=\hbar^{2} 4 \pi \varepsilon_{0} / \mu e^{2} \mathrm{e}$ os coeficientes polinomiais satisfazem à seguinte relação de recursão: $b_{j+1}=\frac{2 Z}{n a} \frac{j+\ell+1-n}{(j+1)(j+2 \ell+2)} b_{j}$.

O coeficiente $b_{0}$ pode ser determinado a partir da condição de normalização do fator radial e, todos os outros, a partir deste. Vale ressaltar que, devido ao polinômio de grau $n-l-1$, o fator radial contém $n-l$-1 zeros (além dos zeros que ocorrem quando $r \rightarrow 0$ e $r \rightarrow \infty$ ). Estes zeros são chamados de zeros (ou nós) internos. A função $R(r)$ também pode ser obtida a partir dos polinômios associados de Laguerre (ref. 4, p. 84):

$$
R_{n, \ell}(r)=-\sqrt{\frac{(n-\ell-1) !}{2 n[(n+\ell) !]^{3}}\left(\frac{2 Z}{n a_{0}}\right)^{3}}\left(\frac{2 Z r}{n a_{o}}\right)^{l} e^{\frac{-Z r}{n a_{0}}} L_{n+\ell}^{2 \ell+1}\left(\frac{2 Z r}{n a_{0}}\right)
$$

em que $\mathrm{a}_{0}$ é o raio de Bohr, dado por $\mathrm{a}_{0}=\mathrm{h}^{2} /\left(4 \pi^{2} \mu \mathrm{e}^{2}\right)$, e $L_{n+\ell}^{2 \ell+1}\left(\frac{2 Z r}{n a_{0}}\right)$ corresponde à $(2 l+1)$-ésima derivada do polinômio de Laguerre de grau $n+l$. O polinômio de Laguerre de um determinado grau $\alpha$ é obtido a partir de (ref. 4, p. 63) $L_{\alpha}(x)=e^{x} \frac{d^{\alpha}}{d x^{\alpha}}\left(x^{\alpha} e^{-x}\right)$.

O fator radial também pode ser expresso em termos de outra função, $P(r)$, definida como $R(r)=\frac{P(r)}{r}$. Com isso a Equação 12 torna-se: 


$$
-\frac{\hbar^{2}}{2 \mu} P^{\prime \prime}(r)+\left[\frac{\ell(\ell+1) \hbar^{2}}{2 \mu r^{2}}+V(r)\right] P(r)=E P(r)
$$

Esta função $P$, que parece ter sido introduzida de forma arbitrária, é de fundamental importância no método de Hartree, principalmente devido à interpretação física associada à mesma, como será visto a seguir.

\section{A função de distribuição radial}

A função de distribuição radial corresponde à densidade de probabilidade de encontrarmos o elétron a uma distância $r$ do núcleo. Podemos obter uma expressão para a mesma a partir da interpretação de Max Born: a probabilidade de encontrarmos o elétron dentro de um elemento de volume $\mathrm{d} \tau$ localizado no ponto $(r, \theta, \phi)$ é dada por $|\Psi|^{2} d \tau \mathrm{Y}$, em que d $\tau$ é dado, em coordenadas esféricas, por $r^{2} \operatorname{sen} \theta d r d \theta d \phi$. Daí segue que

$$
|\Psi|^{2} d \tau=\left[R_{n \ell}(r)\right]^{2}\left|Y_{\ell, m_{\ell}}(\theta, \phi)\right|^{2} r^{2} \operatorname{sen} \theta d r d \theta d \phi
$$

Se quisermos obter a probabilidade de encontrar o elétron em uma casca esférica de raio $r$ e espessura $\mathrm{d} r$ temos que integrar a expressão acima em $\theta$ e em $\phi$ :

$$
\left[R_{n \ell}(r)\right]^{2} r^{2} d r \int_{00}^{2 \pi \pi}\left|Y_{\ell, m_{\ell}}(\theta, \phi)\right|^{2} \operatorname{sen} \theta d r d \theta d \phi=\left[R_{n \ell}(r)\right]^{2} r^{2} d r
$$

pois os harmônicos esféricos são normalizados.

Logo, a função de distribuição radial é dada por $\left[R_{n l}(r)\right]^{2} r^{2}$. Neste ponto vale a pena ressaltar a importância desta função, que pode parecer apenas mais uma expressão matemática. Contudo, é importantíssimo ressaltar que a função de distribuição radial é uma quantidade que pode ser medida experimentalmente. No caso de átomos gasosos, pode ser obtida a partir da técnica de difração de elétrons.6 Os resultados desta técnica revelam a estrutura de camadas $(K, L, M \ldots)$ do átomo, podendo até permitir a estimativa do número de elétrons em cada camada. ${ }^{6}$ Substituindo $R_{n l}(r)$ por $\frac{P_{n \ell}(r)}{r}$ na Equação 16 podemos facilmente verificar que $\left[P_{n l}(r)\right]^{2}$ corresponde à própria função de distribuição radial.

Daqui em diante assumiremos que a massa do elétron é desprezível em relação à massa nuclear, o que leva $\mathrm{a} \mu \approx \mathrm{m}_{\mathrm{e}}$ (massa do elétron). Em geral, esta simplificação representa uma aproximação bastante satisfatória e é necessária, nos átomos multieletrônicos, para evitar complicações adicionais devidas ao chamado efeito de polarização de massa (ref., 5 p. 157-160).

A Equação 14 corresponde à Equação de Schrödinger unidimensional para uma partícula de massa $\mu\left(\approx \mathrm{m}_{\mathrm{e}}\right)$ sujeita a um potencial $V^{\prime}(r)=\frac{\ell(\ell+1) \hbar^{2}}{2 \mu r^{2}}+V(r)$, cujas soluções são dadas pelas funções $P_{n l}(r)$. Consequentemente, estas soluções vão ser tais que, para cada valor de $l$, a $n$-ésima solução possui $n-1$ nós internos (ref. 7, p. 110). Por exemplo, para $l=0$ podemos identificar $\frac{P_{10}(r)}{r}$ como o fator radial de um orbital "do tipo" $1 \mathrm{~s}, \underline{P_{20}(r)}$ como o de um orbital "do tipo" 2 s, e assim por diante. O mesmo raciocínio vale para os outros orbitais. Note que, qualquer que seja a forma funcional de $V(r)$, o número de nós radiais (internos) permanece igual a $n-l-1$, que é o mesmo resultado obtido no caso dos átomos hidrogenoides.

Do que foi dito anteriormente, temos que $\left[P_{n l}(r)\right]^{2} d r$ corresponde à probabilidade de encontrarmos o elétron (no estado caracterizado pelos números quânticos $n$ e $l$ ) numa casca esférica de raio $r$ e espessura $d r$. Classicamente podemos interpretar esta probabilidade como a fração (infinitesimal) da carga total contida na casca esférica, ou $d q=\left[P_{n l}(r)\right]^{2}$, de modo que $\int d q$ corresponde à carga do elétron. Consequentemente, $\left[P_{n l}(r)\right]^{2}$ é a densidade radial de carga. Como veremos logo adiante esta carga infinitesimal $(d q)$ é uma quantidade fundamental nas equações de Hartree.

\section{POTENCIAL ELETROSTÁTICO DE UMA CASCA ESFÉRICA (ref. 8, p. 734)}

Consideremos uma casca esférica de raio $\mathrm{R}$ e carga $Q$. O potencial eletrostático gerado vai depender do ponto em questão. Para um ponto no interior da casca esférica o potencial é constante e dado por $k Q / R$, em que $\mathrm{k}=1 / 4 \pi \varepsilon_{0}$. Para um ponto externo à casca esférica (a uma distância $r$ do seu centro) o potencial é o mesmo que o gerado por uma carga pontual $Q$ localizada no centro da esfera, ou seja, é igual a $k Q / r$ :

$$
V= \begin{cases}\frac{k Q}{r}, & r \geq R \\ \frac{k Q}{R}, & r \leq R\end{cases}
$$

O gráfico de V(r) está mostrado na Figura 1.

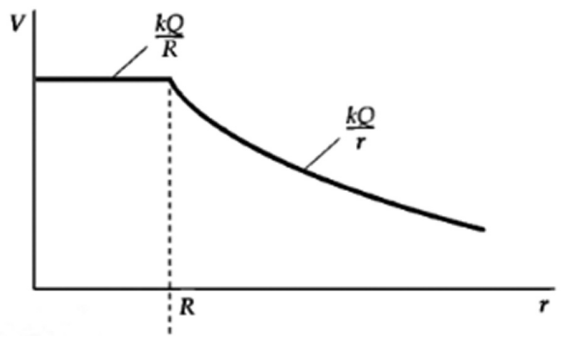

Figura 1. Potencial eletrostático de uma casca esférica de raio $R$

Se a casca esférica possuir espessura infinitesimal $\mathrm{d} r$, então $Q$ pode ser substituído por $\mathrm{d} q$. Como a densidade radial de carga, $\rho(r)$, é dada por $\rho(r)=\frac{d q}{d r} \Rightarrow d q=\rho(r) d r$. Daí segue que, para uma casca esférica, $V(r)=\frac{k \rho(r)}{R} d r$, para $r \leq R, \mathrm{e} V(r)=\frac{k \rho(r)}{r} d r$, para $r \geq R$. Sendo assim, a energia potencial eletrostática que uma dada carga de prova $q$ ' sente é obtida simplesmente multiplicando-se o potencial pela carga, ou seja, é igual a $q$ ' $V$. Nesta etapa é muito importante explicar um aspecto que pode causar confusão. Embora tenhamos utilizado o mesmo símbolo $V$ nas Equações 2, 6, 12 e 14, é importante mencionar que nestas equações $V$ representa a energia potencial eletrostática, e não simplesmente o potencial eletrostático. Porém, quando pensamos no elétron como carga de prova é correto afirmar que o mesmo está submetido (ou sofre a ação de) um potencial.

\section{O PRODUTO DE HARTREE}

Hartree sugeriu que a função de onda eletrônica de um dado átomo contendo $N$ elétrons é dada como um produto de $N$ funções de onda de um elétron:

$$
\begin{aligned}
& \Psi_{H P}\left(r_{1}, \theta_{1}, \phi_{1}, r_{2}, \theta_{2}, \phi_{2}, \cdots, r_{N}, \theta_{N}, \phi_{N}\right) \\
& =u_{n_{1}, \ell_{1}, m_{\ell_{1}}}\left(r_{1}, \theta_{1}, \phi_{1}\right) u_{n_{2}, \ell_{2}, m_{\ell_{2}}}\left(r_{2}, \theta_{2}, \phi_{2}\right) \cdots u_{n_{N}, \ell_{N}, m_{\ell_{N}}}\left(r_{N}, \theta_{N}, \phi_{N}\right)
\end{aligned}
$$


A tríade $\left(r_{i}, \theta_{i}, \phi_{i}\right)$ corresponde às coordenadas esféricas do elétron $i$. Na construção do produto de Hartree $\left(\Psi_{H P}\right)$ assume-se que o princípio da exclusão de Pauli é satisfeito, ou seja, se dois elétrons possuírem os mesmos números quânticos $n, l$ e $m_{l}$ então os números quânticos de spin serão automaticamente diferentes, embora o produto de Hartree não leve em consideração os spins dos elétrons. Os conjuntos dos números quânticos contidos no produto de Hartree é que irão indicar a configuração eletrônica com a qual estamos lidando. Por exemplo, para a configuração $1 \mathrm{~s}^{2} 2 \mathrm{~s}^{1}$ do átomo de Lítio teríamos o seguinte produto de Hartree: $\Psi_{H P}=u_{1,0,0}\left(\mathrm{r}_{1}, \theta_{1}, \phi_{1}\right) u_{1,0,0}\left(\mathrm{r}_{2}, \theta_{2}, \phi_{2}\right) u_{2,0,0}\left(\mathrm{r}_{3}, \theta_{3}, \phi_{3}\right)$.

Uma das principais idéias contidas no método de Hartree diz respeito à escolha das funções monoeletrônicas $(u)$. Ao invés de considerá-las como funções hidrogenoides (o que significaria desprezar completamente as repulsões intereletrônicas), Hartree assumiu que cada uma destas funções é solução de um problema de campo central,

$$
\left[-\frac{\hbar^{2} \nabla_{i}^{2}}{2 m_{e}}+V\left(r_{i}\right)\right] u_{n_{i}, \ell_{i}, m_{\ell i}}\left(r_{i}, \theta_{i}, \phi_{i}\right)=\varepsilon_{n_{i}, \ell_{i}} u_{n_{i}, \ell_{i}, m_{\ell i}}\left(r_{i}, \theta_{i}, \phi_{i}\right)
$$

em que $\varepsilon_{n_{i} l_{i}}$ são os autovalores. O método de Hartree corresponde a uma generalização do problema dos átomos hidrogenoides, pois $V\left(r_{i}\right)$ não é mais um potencial Coulômbico, embora seja esfericamente simétrico. As soluções da Equação 19 são chamadas de orbitais atômicos. Note que as energias (autovalores) dos orbitais não mais dependem apenas do número quântico principal (n), mas também dependem de $l$. Em outras palavras, temos a quebra de degenerescência dos orbitais de um mesmo nível. Esta quebra de degenerescência deve-se ao fato de $\mathrm{V}\left(\mathrm{r}_{\mathrm{i}}\right)$ não ser Coulômbico. Por exemplo, no caso do átomo de $\mathrm{B}\left(1 \mathrm{~s}^{2} 2 \mathrm{~s}^{2} 2 \mathrm{p}^{1}\right)$ os orbitais $2 \mathrm{~s}$ e $2 \mathrm{p}$ não possuem mesma energia.

Por serem soluções de um problema de campo central, cada uma das funções $u$ sempre pode ser expressa como um produto entre um fator radial e um harmônico esférico, ou seja,

$$
u_{n_{i}, \ell_{i}, m_{\ell i}}\left(r_{i}, \theta_{i}, \phi_{i}\right)=R_{n_{i}, \ell_{i}}\left(r_{i}\right) Y_{\ell_{i}, m_{\ell i}}\left(\theta_{i}, \phi_{i}\right)=\left[\frac{P_{n_{i}, \ell_{i}}\left(r_{i}\right)}{r_{i}}\right] Y_{\ell_{i}, m_{\ell i}}\left(\theta_{i}, \phi_{i}\right)
$$

Consequentemente, cada uma das funções $P_{n_{i} l_{i}}\left(r_{i}\right)$ satisfaz à Equação 14':

$$
\begin{gathered}
-\frac{\hbar^{2}}{2 m_{e}} P_{n_{i} \ell_{i}}^{\prime \prime}\left(r_{i}\right)+\left[\frac{\ell_{i}\left(\ell_{i}+1\right) \hbar^{2}}{2 m_{e} r_{i}^{2}}+V\left(r_{i}\right)\right] P_{n_{i} \ell_{i}}\left(r_{i}\right)=\varepsilon_{n_{i} \ell_{i}} P_{n_{i} \ell_{i}}\left(r_{i}\right) \\
\mathrm{i}=1, \ldots, N
\end{gathered}
$$

O grande mérito do método de Hartree consiste na escolha do potencial $V\left(r_{i}\right)$ a que cada um dos elétrons está submetido. Hartree assumiu que cada elétron se move em um potencial esfericamente simétrico (pois $V$ depende apenas da distância elétron-núcleo) devido não apenas ao núcleo, mas também aos outros $N-1$ elétrons. Este potencial geralmente é chamado de campo médio, por levar em conta o efeito de outros elétrons de forma média ou efetiva.

Muitos livros de Físico-Química e Química/Física Quântica explicam o significado físico do campo médio e até mostram a sua expressão matemática, mas não mostram como obtê-lo. Uma das exceções é o livro de Slater (ref. 9, cap. 9). O principal motivo, como já mencionado anteriormente, parece ser a grande ênfase dada ao método de Hartree-Fock, e é no contexto deste método que se costuma explicar o campo médio. É claro que o campo médio representado pelo operador de Fock é que, de fato, é o campo médio correto. Porém, o mesmo contém termos de troca (exchange) que, como dito antes, são devidos à indistinguibilidade dos elétrons e à antissimetria da função de onda eletrônica e não possuem análogo clássico ou, em outras palavras, são o resultado de efeitos puramente quânticos. Por outro lado, o potencial contido no Hamiltoniano efetivo referente ao método de Hartree (Equação 19) pode ser explicado de forma completa utilizando os resultados simples da eletrostática mencionados acima. Sendo assim, acreditamos que começar com o método de Hartree como uma preparação para o método de Hartree-Fock pode representar uma transição mais natural e motivante para o aluno.

\section{OBTENÇÃO DAS EQUAÇÕES DE HARTREE}

Cada elétron $j$ descrito pela função de onda $u_{n_{j} l_{j} m_{l_{l}}}\left(r_{j}, \theta_{j}, \phi_{j}\right)$ gera, no ponto $\left(r_{j}, \theta_{j}, \phi_{j}\right)$, uma densidade de carga igual a $u_{n_{j} l_{j} m_{l j}}^{*}\left(r_{j}, \theta_{j}, \phi_{j}\right)$ $u_{n_{j} l_{j} m_{l j}}\left(r_{j}, \theta_{j}, \phi_{j}\right)$, em unidades da carga do elétron (-e). Consequentemente, a carga $d q_{j}$ contida em um elemento de volume $d \tau_{j}=\left(r_{j}^{2} \operatorname{sen} \theta_{j} d r_{j} d \theta_{j} d \phi_{j}\right)$ centrado em $\left(r_{j}, \theta_{j}, \phi_{j}\right)$ é dada por $d Q_{j}=u_{n_{j} l_{j} m_{l j}}^{*}\left(r_{j}, \theta_{j}, \phi_{j}\right) u_{n_{j} l_{j} m_{l j}}\left(r_{j}, \theta_{j}, \phi_{j}\right) d \tau_{j}$. Para obtermos o chamado campo médio é necessário tornar o potencial gerado por cada elétron esfericamente simétrico, o que é conseguido integrando-se a carga $d Q_{j}$ nas coordenadas angulares $\theta_{j}$ e $\phi_{j}$, ou seja, calculando $\iint_{\theta_{j} \phi_{j}} d Q_{j}=\iint_{\theta_{j} \phi_{j}} u_{n_{j} \ell_{j} m_{\ell j}}^{*}\left(r_{j}, \theta_{j}, \phi_{j}\right) u_{n_{j} \ell_{j} m_{\ell j}}\left(r_{j}, \theta_{j}, \phi_{j}\right) d \tau_{j}=d q_{j}$. Como visto anteriormente, esta integração resulta em (Equações 15 e 16) $d q_{j}=\left[p_{n_{j} l_{j}}\left(r_{j}\right)\right]^{2} d r_{j}$. Além disso, como também vimos, $d q_{j}$ corresponde à carga contida na casca esférica centrada em $r_{j}$ e de espessura igual a $\mathrm{d} r_{j}$. Esta carga é devida ao elétron $j$. O campo médio que um dado elétron $i(\neq j)$ sente é o resultado do potencial esférico gerado pelo núcleo mais o potencial da casca esférica devido a todos os outros elétrons $j$. É desta forma que a energia potencial $\mathrm{V}\left(\mathrm{r}_{\mathrm{i}}\right)$ contida na Equação 14' é obtida.

Sendo assim, o potencial que um dado elétron $i$ localizado a uma distância $r_{i}$ do núcleo sente, devido à casca esférica de outro elétron $j$, é dado por $k \frac{d q_{j}}{r_{j}}=k\left[P_{n_{j} \xi_{j}}\left(r_{j}\right)\right]^{2} \frac{d r_{j}}{r_{j}}$, se $r_{i} \leq r_{j}$, e $k \frac{d q_{j}}{r_{i}}=k\left[P_{n_{j} \xi_{j}}\left(r_{j}\right)\right]^{2} \frac{d r_{j}}{r_{i}}$, se $r_{i} \geq r_{j}$ (Equação 17). Como a casca esférica, na primeira situação $\left(r_{i} \leq r_{j}\right)$, pode estar a uma distância desde $r_{j}=r_{i}$ até $r_{j} \rightarrow \infty$ do núcleo, o potencial devdo a um único elétron $j$ é obtido integrando-se $k\left[P_{n_{j} \ell_{j}}\left(r_{j}\right)\right]^{2} \frac{d r_{j}}{r_{j}}$ de $r_{i}$ a infinito. Além disso, temos que somar o resultado para todos os elétrons $j(\neq i)$. Daí segue que o potencial interno (pois $r_{i} \leq r_{j}$ ) total [que chamaremos que $V_{\text {int }}\left(r_{i}\right)$ ] é dado por

$$
V_{\text {int }}\left(r_{i}\right)=\sum_{j \neq i} \int_{r_{i}}^{\infty} \frac{k\left[P_{n_{j} \ell}\left(r_{j}\right)\right]^{2}}{r_{j}} d r_{j}
$$

De forma análoga, na segunda situação $\left(r_{i} \geq r_{j}\right)$ o potencial devido a um único elétron $j$ é obtido integrando-se $k\left[P_{n_{j} \ell_{j}}\left(r_{j}\right)\right]^{2} \frac{d r_{j}}{r_{i}}$ de 0 a $r_{i}$. Consequentemente, o potencial externo (pois $r_{i} \geq r_{j}$ ) total, devido a todos os elétrons $j(\neq i)\left[V_{\text {ext }}\left(r_{i}\right)\right.$. Obs.: Não confundir este potencial 'externo' com aqueles devidos a perturbações externas, como campo elétrico, magnético, etc...] é dado por

$$
V_{e x t}\left(r_{i}\right)=\sum_{j \neq i} \int_{0}^{r_{i}} \frac{k\left[P_{n_{j} \ell_{j}}\left(r_{j}\right)\right]^{2}}{r_{i}} d r_{j}=\frac{1}{r_{i}} \sum_{j \neq i} \int_{0}^{r_{i}} k\left[P_{n_{j} \ell_{j}}\left(r_{j}\right)\right]^{2} d r_{j}
$$

Lembrando que estes dois potenciais estão expressos em termos da carga do elétron, o potencial total a uma distância $r_{i}$ do núcleo é dado por $k \frac{Z e}{r_{i}}-e\left[V_{\text {int }}\left(r_{i}\right)+V_{e x t}\left(r_{i}\right)\right]$. Logo, a energia potencial de um elétron localizado a esta mesma distância $\left(r_{i}\right)$ do núcleo é obtida simples- 
mente multiplicando-se este potencial total pela carga do elétron, ou seja,

$$
V\left(r_{i}\right)=-k \frac{Z e^{2}}{r_{i}}+e^{2}\left[V_{\text {int }}\left(r_{i}\right)+V_{\text {ext }}\left(r_{i}\right)\right]=V_{\text {nuc }}\left(r_{i}\right)+V_{\text {elec }}\left(r_{i}\right)
$$

em que $V_{\text {nuc }}\left(r_{i}\right)=-k \frac{Z e^{2}}{r_{i}}$ e $V_{\text {elec }}\left(r_{i}\right)=e^{2}\left[V_{\text {int }}\left(r_{i}\right)+V_{\text {ext }}\left(r_{i}\right)\right]$. Esta é exatamente a forma funcional de $V\left(r_{i}\right)\left(\operatorname{com} V_{\text {int }}\left(r_{i}\right)\right.$ e $V_{\text {ext }}\left(r_{i}\right)$ dados pelas Equações 20 e 21, respectivamente) que deve ser utilizada nas Equações 14' a fim de obtermos as funções $\mathrm{P}$ dos orbitais atômicos.

Note que para obtermos a função $P$ de um dado elétron $i$ precisamos de $V\left(r_{i}\right)$, e para obtermos $V\left(r_{i}\right)$ é necessário dispormos das funções $P$ de todos os outros elétrons (Equações 14', 20, 21 e 22). Por outro lado, para obtermos as funções $P$ de todos os outros elétrons temos que resolver o conjunto de Equações 14' para $j \neq i$, o que requer o conhecimento de $V\left(r_{j}\right)$ que, por sua vez, requer o conhecimento da função $P$ do elétron $i$. Consequentemente, a solução das Equações 14 ' requer um procedimento iterativo, também chamado de método das aproximações sucessivas. Neste procedimento escolhe-se um conjunto inicial de funções $P$ (uma para cada elétron) e, em seguida, forma-se a função $V\left(r_{i}\right)$ para cada elétron $i$. Um "chute" inicial razoável corresponde às funções $P$ dos orbitais hidrogenoides, com um número atômico efetivo adequadamente escolhido para cada elétron. Estes números atômicos efetivos podem ser escolhidos, por exemplo, segundo as regras de Slater (ref. 4, p. 163). De posse de $V\left(r_{i}\right)$ resolve-se o conjunto das Equações 14' e obtém-se um novo conjunto de funções $P$, juntamente com os seus autovalores (energias dos orbitais). O procedimento é repetido até que as autofunções e os autovalores obtidos na etapa (ou passo) anterior coincidam (dentro de uma tolerância pré-definida) com os obtidos no passo atual. Quando isso ocorre dizemos que foi atingida a convergência. Este tipo de procedimento iterativo foi sugerido por Hartree, e é chamado de método do campo autoconsistente (self-consistent field), simplesmente denominado de método SCF. Vale ressaltar que o procedimento SCF é inerente tanto ao método de Hartree quanto ao método de Hartree-Fock. Os detalhes deste procedimento estão muito bem explicados no livro de Slater (ref. 9, p. 220-222 ) e no livro de Hartree (ref. 10, cap. 4 e 5).

Como esperado, $V\left(r_{i}\right)$ tende a $-k \frac{Z e^{2}}{r_{i}}$ quando $r_{i} \rightarrow 0$, e tende a $-k \frac{e^{2}}{r_{i}}$ quando $r_{i} \rightarrow \infty$. Ou seja, quando o elétron está muito perto do núcleo ele sente a carga nuclear completamente desblindada, e quando está muito longe sente a carga nuclear completamente blindada pelos outros elétrons.

\section{O HAMILTONIANO ATÔMICO EFETIVO $\left(\hat{H}_{e f}\right)$ COMO PONTO DE PARTIDA PARA O TRATAMENTO DA PERTURBAÇÃO DEVIDO À REPULSÃO INTERELETRÔNICA}

A partir da definição do produto de Hartree ( $\Psi_{H P}$, Equação 18) e da Equação 19, podemos verificar facilmente que $\Psi_{H P}$ é solução da equação $\hat{H}_{e f} \Psi_{H P=} \varepsilon \Psi_{H P}$, em que $\hat{H}_{e f}=\sum_{i=1}^{N}\left\lfloor-\frac{\hbar^{2} \nabla_{i}^{2}}{2 m_{e}}+V\left(r_{i}\right)\right\rfloor$ e $\varepsilon=\varepsilon_{n_{1} \ell_{1}}+\varepsilon_{n_{2} \ell_{2}}+\cdots+\varepsilon_{n_{N} \ell_{N}}$. Lembrando que $V\left(r_{i}\right)=-k \frac{Z e^{2}}{r_{i}}+e^{2}\left[V_{\text {int }}\left(r_{i}\right)+V_{\text {ext }}\left(r_{i}\right)\right]$ (Equação 22) podemos observar que $\hat{H}_{e f}$ não é dado simplesmente pela soma de Hamiltonianos hidrogenoides (com carga Z), $\hat{H}_{0}=\sum_{i=1}^{N}\left[-\frac{\hbar^{2} \nabla_{i}^{2}}{2 m_{e}}-\frac{k Z e^{2}}{r_{i}}\right]$, mas sim pela soma de Hamiltonianos efetivos de uma partícula. De acordo com a análise feita na seção anterior já sabemos que o termo $e^{2}\left[V_{i n t}\left(r_{i}\right)+V_{\text {ext }}\left(r_{i}\right)\right]$ em $V\left(r_{i}\right)$ inclui, no potencial do elétron $i$, o efeito (médio) da repulsão eletrônica devida aos outros elétrons. Lembrando também que o Hamiltoniano com- pleto do átomo (desprezando o efeito de polarização de massa e o acoplamento spin-órbita) é dado por $\hat{H}=\sum_{i=1}^{N}\left[-\frac{\hbar^{2} \nabla_{i}^{2}}{2 m_{e}}-\frac{k Z e^{2}}{r_{i}}+\sum_{i>j} \frac{k e^{2}}{r_{i j}}\right]$, temos $\hat{H}-\hat{H}_{e f}=\hat{H}^{\prime}=\sum_{i>j} \frac{k e^{2}}{r_{i j}}-e^{2}\left[V_{\text {int }}\left(r_{i}\right)+V_{e x t}\left(r_{i}\right)\right]$ em que definimos $\hat{H}^{\prime}$ como a perturbação causada pela repulsão intereletrônica instantânea. Note que poderíamos ter definido a perturbação $\hat{H}^{\prime}$ como $\hat{H}^{\prime}=\hat{H}-\hat{H}_{0}=\sum_{i>j} \frac{k e^{2}}{r_{i j}}$. Porém, salta aos olhos que a perturbação definida da forma anterior é menor que a definida desta forma. Consequentemente, os resultados obtidos com aquela serão melhores.

Embora o Hamiltoniano efetivo aqui apresentado não seja o mais correto (pois despreza os termos de troca, que são incluídos no Hamiltoniano de Fock), o objetivo desta análise é mostrar a importância da repulsão intereletrônica média como um ponto de partida mais adequado do que aquele em que este tipo de repulsão é desprezada completamente. Como seria de se esperar, um ponto de partida ainda mais adequado seria o Hamiltoniano de Fock (ref. 11, seção 6.5).

\section{CARGA NUCLEAR EFETIVA $\left(Z_{\mathrm{ef}}\right)$} como

Podemos reescrever a Equação 22, $V\left(r_{i}\right)=-k \frac{Z e^{2}}{r_{i}}+e^{2}\left[V_{\text {int }}\left(r_{i}\right)+V_{\text {ext }}\left(r_{i}\right)\right]$,

$$
V\left(r_{i}\right)=-\frac{k e^{2}}{r_{i}}\left\{Z-\frac{r_{i}}{k}\left[V_{\text {int }}\left(r_{i}\right)+V_{e x t}\left(r_{i}\right)\right]\right\}
$$

Daí segue que podemos definir a carga nuclear efetiva $\left(Z_{e f}\right)$ como

$Z_{e f}=Z-r_{i} \frac{\left[V_{\text {int }}\left(r_{i}\right)+V_{e x t}\left(r_{i}\right)\right]}{k}=Z-\left\{r_{i} \sum_{j \neq i}^{\infty}\left[\frac{\left[\mathrm{P}_{\mathrm{n}_{j} j}\left(r_{j}\right)\right]^{2} d r_{j}}{r_{j}}+\sum_{j \neq i} \int_{0}^{r_{i}}\left[\mathrm{P}_{\mathrm{n}_{j}, j}\right]\left(r_{j}\right)^{2} d r_{j}\right\}\right.$

Como resultado do comportamento de $V_{\text {elec }}\left(r_{i}\right)=e^{2}\left[V_{\text {int }}\left(r_{i}\right)+V_{\text {ext }}\left(r_{i}\right)\right]$, $Z_{e f}$ tem a propriedade de $\operatorname{Lim} Z_{e f}=Z$ e $\operatorname{Lim} Z_{e f}=1$, como seria de se esperar. Para um exemplo do comportamento de $Z_{e f}\left(r_{i}\right)$ ver ref. 9, p. 228. Para maiores detalhes recomendamos o artigo de Duarte, ${ }^{12}$ no qual o conceito de carga nuclear efetiva é discutido de forma extremamente clara.

\section{FORMA ALTERNATIVA PARA AS EQUAÇÕES DE HARTREE}

Sabendo que $V\left(r_{i}\right)=-k \frac{Z e^{2}}{r_{i}}+e^{2}\left[V_{\text {int }}\left(r_{i}\right)+V_{\text {ext }}\left(r_{i}\right)\right]=V_{\text {nuc }}\left(r_{i}\right)+V_{\text {elec }}\left(r_{i}\right)$ (Equação 22), a Equação 19 pode ser reescrita como:

$\left[-\frac{\hbar^{2} \nabla_{i}^{2}}{2 m_{e}}-k \frac{Z e^{2}}{r_{i}}+V_{\text {elec }}\left(r_{i}\right)\right] u_{n_{i}, \ell_{i}, m_{i i}}\left(r_{i}, \theta_{i}, \phi_{i}\right)=\varepsilon_{n_{i}, \ell_{i}} u_{n_{i}, \ell_{i}, m_{i i}}\left(r_{i}, \theta_{i}, \phi_{i}\right)$

Pode-se mostrar, utilizando a expansão de $1 / r_{i j}$ em termos de harmônicos esféricos (ref. 4, p. 369) juntamente com a 'média esférica' sobre as coordenadas do elétron $i$ (ver apêndice), que $\sum_{j \neq i} \int\left|u_{n_{j} \ell_{m} m_{j j}}\right|^{2} \frac{k e^{2}}{r_{i j}} d \tau_{j}=V_{\text {elec }}\left(r_{i}\right)=e^{2}\left[V_{\text {int }}\left(r_{i}\right)+V_{\text {ext }}\left(r_{i}\right)\right]$, em que os dois termos do lado direito são dados pelas Equações 20 e 21, respectivamente. Com isso, a Equação 19' pode ser reescrita da seguinte forma:

$\left[-\frac{\hbar^{2} \nabla_{i}^{2}}{2 m_{e}}-k \frac{Z e^{2}}{r_{i}}+\sum_{j \neq i} \int\left|u_{n_{j} \ell_{j} m_{\ell_{j}}}\right|^{2} \frac{k e^{2}}{r_{i j}} d \tau_{j}\right] u_{n_{i}, \ell_{i}, m_{i i}}\left(r_{i}, \theta_{i}, \phi_{i}\right)=\varepsilon_{n_{i}, \ell_{i}} u_{n_{i}, \ell_{i}, m_{i j}}\left(r_{i}, \theta_{i}, \phi_{i}\right)$

em que $i=1, \ldots, N$. Esta é a forma na qual as equações de Hartree são apresentadas em vários livros como, por exemplo, ref. 3 (seção 
11.1), ref. 4 (p. 164), ref. 13 (seção 7.3) e ref. 14 (p. 175). A grande vantagem de apresentá-las desta forma está na identificação quase que imediata dos três termos do lado esquerdo: o primeiro se refere à energia cinética do elétron $i$, o segundo se refere à atração elétronnúcleo, enquanto que o terceiro expressa a repulsão eletrostática média devida aos outros elétrons. Vale lembrar que, uma vez que

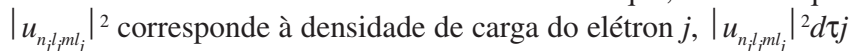
fornece a fração da carga do elétron $j$ contida no volume $d \tau_{j}$. Assim, $k e^{2} \frac{\left|u_{n_{j} \ell_{m} m_{j j}}\right|^{2}}{r_{i j}} d \tau_{j}$ fornece a energia potencial eletrostática que o elétron $i$ (localizado em $r_{i}$ ) sente como resultado da presença do elétron $j$ (localizado em $r_{j}$ ). A integração nas coordenadas do elétron $j$ visa levar em conta todas as possíveis localizações do mesmo. Assim, esta integração nos fornece a repulsão intereletrônica média que o elétron $i$ sente (como resultado da presença do elétron $j$ ), em oposição à repulsão intereletrônica instantânea, contida no Hamiltoniano atômico completo. Somando as contribuições devidas a todos os elétrons $j(\neq i)$ obtemos a repulsão intereletrônica média total.

Utilizando a Equação 23 juntamente com o resultado obtido no apêndice, $\left[V_{\text {int }}\left(r_{i}\right)+V_{\text {ext }}\left(r_{i}\right)\right]=\sum_{j \neq i} \int\left|u_{n_{j}, m l_{j}}\right|^{2} \frac{k}{r_{i j}} d \tau_{j}$, podemos expressar a carga efetiva que um dado elétron $i$ sente (a uma distância $r_{i}$ do núcleo) a partir dos termos de atração elétron-núcleo e da repulsão eletrostática média devida aos outros elétrons:

$$
-\frac{k e^{2} Z_{e f}}{r_{i}}=V_{n u c}+\sum_{j \neq i} \int\left|u_{n_{j} \ell_{j} m_{\ell_{j}}}\right|^{2} \frac{k e^{2}}{r_{i j}} d \tau_{j}
$$

Esta equação nos permite chegar a uma interpretação bastante clara a respeito do significado físico da carga efetiva. Analisando-a podemos dizer que a carga efetiva, para um elétron a uma distância $r_{i}$ do núcleo, corresponde à carga que o mesmo deve ter a fim de que a sua energia potencial seja igual à soma entre a energia de atração elétron-núcleo e a energia de repulsão eletrostática média devida aos outros elétrons. Baseando-se nesta ideia Hartree apresentou resultados referentes às cargas efetivas, em função da distância elétronnúcleo, para os elétrons nas várias subcamadas dos íons $\mathrm{Cl}^{-} \mathrm{e} \mathrm{Cu}^{+}$.

\section{OBTENÇÃO DAS EQUAÇÕES DE HARTREE A PARTIR DO MÉTODO VARIACIONAL}

Slater (ref. 9, seção 9.4 e ref. 16) e Fock ${ }^{17}$ mostraram como obter as Equações de Hartree (Equação 14', com $V\left(r_{\mathrm{i}}\right)$ dado pelas Equações 20, 21 e 22) a partir do método variacional, utilizando as seguintes etapas: (i) calcula-se o valor esperado da energia, utilizando o produto de Hartree como função de onda tentativa; (ii) em seguida, é feita a média angular deste valor esperado, ou seja, o mesmo é integrado em relação às coordenadas $\theta$ e $\phi$ de cada par de elétrons. Esta integração é consistente com o fato de a parte angular dos orbitais ser dada pelos harmônicos esféricos, caracterizando assim o potencial esferossimétrico, como visto anteriormente; (iii) esta energia é então minimizada em relação aos orbitais (na realidade, em relação à parte radial dos mesmos, pois a parte angular já é conhecida), o que leva às Equações de Hartree. Esta demonstração mais rigorosa foi muito importante para dar um maior suporte ao método de Hartree, pois até meados de 1930 ainda não estava claro o suficiente se os orbitais de Hartree eram de fato as melhores funções monoeletrônicas do ponto de vista variacional, ou seja, que levam à menor energia para uma função cuja forma é restrita ao produto de Hartree e com a parte angular dada pelos harmônicos esféricos. Além disso, esta demonstração serviu de base para a obtenção das Equações de Hartree-Fock, realizada logo em seguida.

\section{O TEOREMA DE ÜNSOLD COMO JUSTIFICATIVA PARA O MÉTODO DE HARTREE}

Apesar da repulsão intereletrônica instantânea que um dado elétron sente (devido à presença dos outros) não ser esfericamente simétrica, caso os outros elétrons formem uma subcamada semipreenchida ou completamente preenchida, a densidade eletrônica devida aos mesmos é esfericamente simétrica, um resultado conhecido como teorema de Ünsold, ${ }^{18}$ ou seja,

$$
2 \sum_{m_{\ell}=-\ell}^{\ell}\left|R_{n, \ell}(r)\right|^{2}\left|Y_{\ell, m_{\ell}}(\theta, \phi)\right|^{2}=2\left|R_{n, \ell}(r)\right|^{2} \sum_{m_{\ell}=-\ell}^{\ell}\left|Y_{\ell, m_{\ell}}(\theta, \phi)\right|^{2}=\frac{(2 \ell+1)}{2 \pi}\left|R_{n, \ell}(r)\right|^{2}
$$

$\mathrm{O}$ fator 2 deve-se à subcamada completamente preenchida. Se a mesma for semipreenchida o fator 2 deve ser substituído pelo número 1. Sendo assim, o teorema de Ünsold pode ser considerado como uma justificativa (ainda que parcial) para a presença de um potencial esferossimétrico devido aos elétrons.

\section{ENERGIA TOTAL DO ÁTOMO}

A energia total do átomo, resultante da aproximação de Hartree, pode ser obtida simplesmente calculando-se o valor esperado da energia com a função de onda na forma do produto de Hartree, ou seja, $E=\int \Psi_{H P}^{*} \hat{H} \Psi_{H P} d \tau$, o que resulta em (ref. 3, p. 312 ; ref. 4, p. 165):

$$
E=\sum_{i=1}^{N} \varepsilon_{i}-\sum_{i=1}^{N} \sum_{j>i} J_{i j}
$$

em que $J_{i j}=\iint \frac{\left|u_{n_{i}, m_{\ell_{i}}}\right|^{2}\left|u_{n_{\ell^{\prime} \ell_{j} m_{j}}}\right|^{2}}{r_{i j}} d \tau_{i} d \tau_{j}$ e $\varepsilon_{\mathrm{i}}=\varepsilon_{n_{i} l_{i} m l_{i}}$. Como podemos observar a partir da Equação 25, a energia total do átomo não é simplesmente a soma das energias dos orbitais, mas inclui também a repulsão Coulômbica $\left(J_{i j}\right)$ entre as nuvens eletrônicas de todos os pares de elétrons.

Embora o produto de Hartree, $\psi_{\mathrm{HP}}$, não seja uma função de onda qualitativamente correta para representar os termos (ou estados) espectroscópicos [pois a mesma não é, no caso mais geral, autofunção dos operadores $\hat{S}^{2}$ e $\hat{L}^{2}$, descaracterizando assim os termos espectroscópicos no esquema LS, ou Russel-Saunders (ref. 4, cap. 9; ref. 9, cap. 10)], ela serve para associarmos os termos espectroscópicos às configurações eletrônicas que os originaram. Isto se deve ao fato de as energias das configurações, que seriam degeneradas caso desprezássemos completamente a repulsão intereletrônica, passarem a ser diferentes quando levamos em conta esta repulsão, ainda que de forma média. Por exemplo, as configurações $1 \mathrm{~s}^{2} 2 \mathrm{~s}^{1}$ e $1 \mathrm{~s}^{2} 2 \mathrm{p}^{1}$ do átomo de lítio possuem energias diferentes, tanto devido à quebra da degenerescência entre os orbitais $2 \mathrm{~s}$ e $2 \mathrm{p}$ quanto devido ao fato de as integrais $J_{1 \mathrm{~s} 2 \mathrm{~s}}$ e $J_{1 \mathrm{~s} 2 \mathrm{p}}$ serem diferentes (ref. 3, p. 271-272).

Vale ressaltar que as funções de onda qualitativamente corretas que descrevem os termos espectroscópicos são formadas a partir de combinações lineares de determinantes de Slater (ref. 4, seções 9d a 9f). Cada microestado da configuração eletrônica (isto é, cada forma de distribuirmos os elétrons nos orbitais que definem a configuração eletrônica) corresponde a um determinante de Slater. Por exemplo, há 12 microestados associados à configuração $1 \mathrm{~s}^{1} 2 \mathrm{p}^{1}$ (ref. $4, \mathrm{p}$. 132) e 15 associados à configuração $1 \mathrm{~s}^{2} 2 \mathrm{~s}^{2} 2 \mathrm{p}^{2}$ (ref. 4, p. 140-141; ref. 5, p. 264-265). Sendo assim, no primeiro caso há 12 determinantes de Slater, enquanto que no segundo há 15 . 


\section{ALGUNS RESULTADOS OBTIDOS COM O MÉTODO DE HARTREE}

\section{Energias de ionização do He e energias dos níveis de raios-X do $\mathbf{R b}^{+}$}

Hartree $^{2}$ verificou uma excelente concordância entre o valor calculado $(24,84 \mathrm{eV})$ para a energia do orbital 1s do átomo de $\mathrm{He}$ no estado fundamental e o valor experimental obtido para a primeira energia de ionização (24,6 eV). Esta concordância pode ser devida a qualquer uma das duas situações: os efeitos de relaxação orbital (ou seja, o rearranjo da nuvem eletrônica como resultado da ionização) e correlação eletrônica são muito pequenos ou, estes dois efeitos se cancelam. Como a relaxação orbital certamente não é pequena neste caso, ${ }^{2} \mathrm{o}$ mais provável é que neste caso os dois efeitos mencionados se cancelam. Hartree menciona nesse mesmo artigo que a decisão de testar o seu método no átomo de hélio foi consequência dos bons resultados obtidos para as energias dos níveis de raios- $\mathrm{X}$ de outros átomos ou íons. Por exemplo, no caso do $\mathrm{Rb}^{+}$a concordância entre os valores calculados para as energias dos orbitais mais internos (referentes às camadas $\mathrm{K}, \mathrm{L}, \mathrm{M}$ e N) e as energias dos níveis de raios-X é muito boa, com erro máximo de $\sim 8 \%$. $^{2}$

\section{Funções de distribuição radial para alguns átomos ou íons com configuração eletrônica de gás nobre}

Na Figura 2 estão mostrados os gráficos das funções de distribuição radial [denominadas de $\mathrm{D}(\mathrm{r})$ no gráfico] calculadas com o método de Hartree, ${ }^{2}$ para o $\mathrm{He}, \mathrm{Na}^{+}$e $\mathrm{Rb}^{+}$.

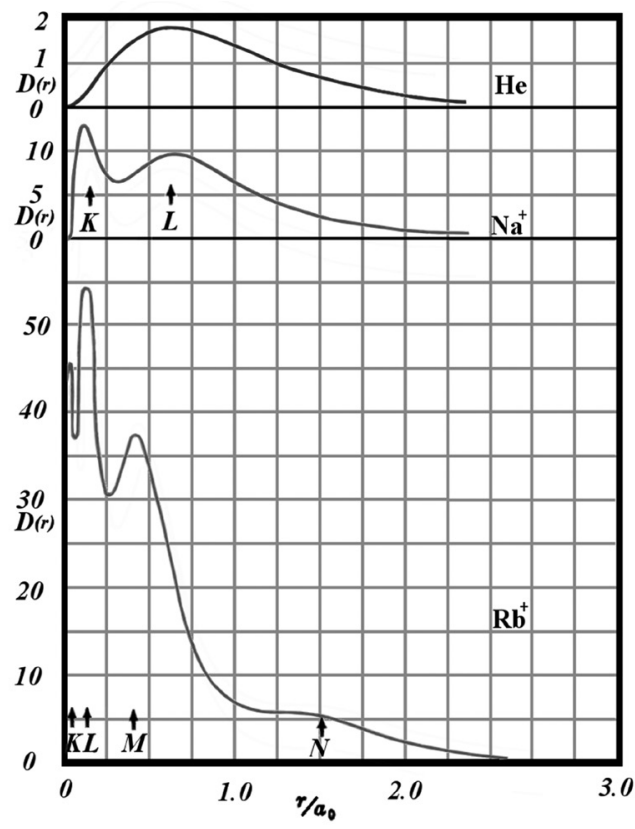

Figura 2. Função de distribuição radial obtida a partir do método de Hartree ${ }^{2}$ (adaptada da ref. 4), para o $\mathrm{He}, \mathrm{Na}^{+}$e $\mathrm{Rb}^{+}$. A área sob a curva fornece o número total de elétrons do átomo

É bastante instrutivo ressaltar que as camadas eletrônicas podem ser claramente identificadas, e ocorrem de acordo com o que é esperado a partir das configurações eletrônicas das espécies estudadas. Além disso, também podemos notar que o 'raio' destas camadas diminui à medida que $Z$ aumenta, em concordância com o que se espera a partir do aumento da carga nuclear efetiva ${ }^{12}$ que os elétrons destas camadas sentem. Por exemplo, o raio da camada $K\left(r_{k}\right)$ é dado, aproximadamente, pela expressão $r_{K}=a 0 / 2 \mathrm{Z}$, em que $\mathrm{a}_{0}$ é o raio de Bohr. Tal resultado é consistente com fato de a camada $K$, tendo um número constante de elétrons, sentir uma atração cada vez maior da carga nuclear crescente. Outros resultados referentes às funções de distribuição radial, para os íons $\mathrm{Cu}^{+}, \mathrm{K}^{+} \mathrm{e} \mathrm{Rb}^{+}$(incluindo as contribuições das subcamadas mais externas) podem ser encontrados na ref. 15. Na ref. 19 as funções de distribuição radial das espécies O, $\mathrm{O}^{+}, \mathrm{O}^{++}$e $\mathrm{O}^{+++}$são comparadas.

No mesmo artigo de onde foram retirados os resultados mostrados na Figura $2,{ }^{2}$ Hartree comenta que os fatores $\mathrm{F}$ (também chamado de fator de estrutura atômico) obtidos a partir das funções de distribuição radial dos íons $\mathrm{Na}^{+} \mathrm{e} \mathrm{Cl}^{-}$levam a uma boa concordância entre o difratograma de raios-X calculado e o medido experimentalmente para o $\mathrm{NaCl}(\mathrm{s})$.

\section{CONCLUSÕES}

No ensino de Química Quântica sugerimos apresentar o método de Hartree antes do de Hartree-Fock, pois aquele já contém aspectos importantes que resultam da repulsão intereletrônica, como o conceito de orbitais atômicos em um ambiente multieletrônico, quebra da degenerescência dos orbitais de mesmo $l$ (por exemplo, $2 \mathrm{p}>2 \mathrm{~s}$ ) e energia de uma configuração. Além disso, o método de Hartree representa uma ótima oportunidade de unir conceitos de Física (no caso específico, da eletrostática) com os de Química Quântica (no caso do método de Hartree, o de campo central), o que pode contribuir para diminuir o gap que há entre o ensino de Física e o de Química. Acreditamos também que o método de Hartree, por ser o primeiro método autoconsistente em estrutura eletrônica de átomos e por conter conceitos mais fáceis de assimilar que os contidos no método de Hartree-Fock (como a indistinguibilidade das partículas quânticas e a antissimetria da função de onda), pode servir como uma boa preparação, do ponto de vista didático, para este.

Para enriquecer um pouco mais o tema, é interessante apresentar para o aluno a cronologia dos trabalhos seminais relacionados a estes dois métodos. Cerca de 1 ano após a publicação dos três primeiros artigos relacionados ao método de Hartree, ${ }^{1,2,20}$ Slater mostrou a forma funcional da função de onda mais simples possível que satisfaz aos princípios da indistinguibilidade e da antissimetria, o chamado determinante de Slater. No ano seguinte, Fock, de posse do know-how adquirido na obtenção das Equações de Hartree via método variacional, ${ }^{17}$ aplicou, nesse mesmo artigo, o método variacional à função de onda tentativa dada na forma de determinante de Slater e deduziu as equações hoje conhecidas como Equações de Hartree-Fock (porém, o método foi inicialmente denominado, pelo próprio Fock, de método de Hartree generalizado, ao invés de método de Hartree-Fock, como hoje o denominamos). Em seguida, as equações referentes ao átomo de sódio foram deduzidas, ${ }^{22}$ mas ainda sem a apresentação de resultados numéricos. Vale ressaltar que apenas quase 4 anos após a obtenção destas equações é que foram apresentados os primeiros resultados obtidos a partir da resolução numérica das mesmas, para este mesmo átomo. ${ }^{23}$ Segundo o próprio Fock, esta demora foi consequência da forma destas equações, que tornavam os métodos de integração numérica usuais inaplicáveis. Sendo assim, foi necessário propor um novo método de solução numérica que fosse aplicável às equações generalizadas do campo autoconsistente (isto é, às Equações de Hartree-Fock ${ }^{23}$ ). Este fato ilustra muito bem o papel essencial que os métodos numéricos desempenham no desenvolvimento dos métodos computacionais em Química Quântica.

\section{AGRADECIMENTOS}

Às agências de fomento CNPq (Edital MCT/CNPq No 06/2008 - Jovens Pesquisadores e Bolsa de Produtividade) e CAPES pelo auxílio financeiro. 


\section{APENNDICE}

Nesta seção iremos demonstrar, tomando a média esférica em relação às coordenadas do elétron $i$ (como requer o modelo do campo central), que

$\sum_{j \neq i} \int\left|u_{n_{j} \ell_{j} m_{\ell_{j}}}\right|^{2} \frac{k e^{2}}{r_{i j}} d \tau_{j}=\sum_{j \neq i} \int_{\eta_{i}}^{\infty} \frac{k e^{2}\left[P_{n_{j} \ell_{j}}\left(r_{j}\right)\right]^{2}}{r_{j}} d r_{j}+\frac{1}{r_{j}} \sum_{j \neq i} \int_{0}^{r_{i}} k e^{2}\left[P_{n_{j} \ell_{j}}\left(r_{j}\right)\right]^{2} d r_{j}$

O potencial eletrostático devido ao elétron $j$, na posição do elétron $i$, é dado por $\left|u_{n_{j j} m_{\ell_{j}}}\right|^{2} \frac{k e}{r_{i j}} d \tau_{j}$. A integração nas coordenadas angula res do elétron $j$ nos permite obter o potencial eletrostático devido à casca esférica (de raio $r_{j}$ ) resultante da carga do elétron $j$, na posição do elétron $i$. Esta 'média esférica' é consistente com o modelo do campo central. Visando levar em conta todos os raios possíveis da casca esférica do elétron $j$ também devemos integrar esta expressão em $r_{j}$, o que resulta em:

$$
k e \int_{0}^{\infty}\left[\int_{0}^{2 \pi \pi} \int_{0}^{\left|u_{n_{j} m_{\ell_{j}}}\right|^{2}} r_{i j} \Omega_{j}\right] r_{j}^{2} d r_{j}
$$

em que $d \Omega_{\mathrm{j}}=\operatorname{sen} \theta_{j} d \theta_{j} d \phi_{j}$. Utilizando a expansão de $1 / \mathrm{r}_{\mathrm{ij}}$ em harmônicos esféricos (ref. 4 , p. 369)

$$
\frac{1}{r_{i j}}=\sum_{\ell=0}^{\infty} \sum_{m_{\ell}=-\ell}^{m_{\ell}=\ell} \frac{4 \pi}{(2 \ell+1)} Y_{\ell, m_{\ell}}\left(\theta_{i}, \phi_{i}\right) Y_{\ell, m_{\ell}}^{*}\left(\theta_{j}, \phi_{j}\right) \frac{r_{<}^{\ell}}{r_{>}^{\ell+1}}
$$

( $r_{<}$e $r_{>}$correspondem, respectivamente, ao menor e ao maior entre os valores de $r_{i}$ e $r_{j}$ ), e substituindo na expressão anterior obtemos

$4 \pi k e \sum_{\ell} \sum_{m_{\ell}} \frac{1}{(2 \ell+1)} Y_{\ell, m_{\ell}}\left(\theta_{i}, \phi_{i}\right) \int_{0}^{\infty}\left[\int_{0}^{2 \pi \pi} \int_{0}^{2 \pi}\left|u_{n_{j} \xi_{j} m_{j}}\right|^{2} Y_{\ell, m_{\ell}}^{*}\left(\theta_{j}, \phi_{j}\right) d \Omega_{j}\right] \frac{r_{<}^{\ell}}{r_{>}^{\ell+1}} r_{j}^{2} d r_{j}$

Como podemos observar, este potencial é independente da direção do raio vetor do elétron $j$. Para torná-lo independente das direções dos dois elétrons ( $i$ e $j$ ) devemos integrar esta expressão nas coordenadas angulares do elétron $i$ e, para fins de normalização, dividir o resultado por $4 \pi$ (esta abordagem também é considerada na ref. 3 , p. 311), o que resulta em:

$k e \sum_{\ell} \sum_{m_{\ell}} \frac{1}{(2 \ell+1)} \int Y_{\ell, m_{\ell}}\left(\theta_{i}, \phi_{i}\right) d \Omega_{i} \int_{0}^{\infty}\left[\int_{0}^{2 \pi \pi} \iint_{0}^{2}\left|u_{n_{j} \ell_{j} m_{\ell_{j}}}\right|^{2} Y_{\ell, m_{\ell}}^{*}\left(\theta_{j}, \phi_{j}\right) d \Omega_{j}\right] \frac{r_{<}^{\ell}}{r_{>}^{\ell+1}} r_{j}^{2} d r_{j}$

Porém, sabemos que $Y_{0,0}\left(\theta_{i}, \phi_{i}\right)=1 / \sqrt{ } 4 \pi=Y_{0,0}\left(\theta_{j}, \phi_{j}\right)$. Logo, $Y_{0,0}^{*}$ $\left(\theta_{i}, \phi_{i}\right) Y_{0,0}\left(\theta_{j}, \phi_{j}\right)=1 / 4 \pi$. Com isso a expressão anterior se torna

$\left.\left.k e \sum_{\ell} \sum_{m_{\ell}} \frac{1}{(2 \ell+1)} \int Y_{0,0}^{*}\left(\theta_{i}, \phi_{i}\right) Y_{\ell, m_{\ell}}\left(\theta_{i}, \phi_{i}\right) d \Omega_{i} \int_{0}^{\infty} \int_{0}^{2 \pi \pi} \int_{0}^{2 \pi} \int_{0} u_{n_{j} \ell, m_{\ell}}\right|^{2} Y_{\ell, m_{\ell}}^{*}\left(\theta_{j}, \phi_{j}\right) Y_{0,0}\left(\theta_{j}, \phi_{j}\right) d \Omega_{j}\right] \frac{r_{\ell}^{\ell}}{r_{>}^{\ell+1}} r_{j}^{2} d r_{j}$

Devido à ortonormalidade entre os harmônicos esféricos obtemos $4 \pi k e \sum_{\ell} \sum_{m_{\ell}} \frac{1}{(2 \ell+1)} \delta_{\ell, 0} \delta_{m_{\ell}, 0} \int_{0}^{\infty}\left[\int_{0}^{2 \pi} \int_{0}^{\pi}\left|u_{n_{j} \ell_{j} m_{\ell_{j}}}\right|^{2} Y_{\ell, m_{\ell}}^{*}\left(\theta_{j}, \phi_{j}\right) Y_{0,0}\left(\theta_{j}, \phi_{j}\right) d \Omega_{j}\right] \frac{r_{<}^{\ell}}{r_{>}^{\ell+1}} r_{j}^{2} d r_{j}$ o que resulta em

$4 \pi k e \int_{0}^{\infty}\left[\int_{0}^{2 \pi} \int_{0}^{\pi}\left|u_{n, \ell_{j}, m_{\ell_{j}}}\right|^{2} Y_{0,0}^{*}\left(\theta_{j}, \phi_{j}\right) Y_{0,0}\left(\theta_{j}, \phi_{j}\right) d \Omega_{j}\right] \frac{1}{r_{>}} r_{j}^{2} d r_{j}=k e \int_{0}^{\infty}\left[\int_{0}^{2 \pi} \int_{0}^{\pi}\left|u_{n_{\ell}, j_{j} m_{j}}\right|^{2} d \Omega_{j}\right] \frac{1}{r_{>}} r_{j}^{2} d r_{j}$

Uma vez que $\left|u_{n_{j} \ell_{j} m_{\ell_{j}}}\right|^{2}=\frac{\left[P_{n_{j} \ell_{j}}\left(r_{j}\right)\right]^{2}}{r_{j}^{2}}\left|Y_{\ell_{j} m_{\ell_{j}}}\left(\theta_{j}, \phi_{j}\right)\right|^{2}$ obtemos o seguinte resultado:

$k e \int_{0}^{\infty}\left[\int_{0}^{2 \pi \pi} \int_{0}^{2 \pi}\left|Y_{\ell_{j} m_{\ell_{j}}}\left(\theta_{j}, \phi_{j}\right)\right|^{2} d \Omega_{j}\right] \frac{\left[P_{n_{j} \ell_{j}}\left(r_{j}\right)\right]^{2}}{r_{>}} d r_{j}=k e \int_{0}^{\infty} \frac{\left[P_{n_{j} \ell_{j}}\left(r_{j}\right)\right]^{2}}{r_{>}} d r_{j}$, devido à normalização dos harmônicos esféricos. Os limites de integração podem ser subdivididos em dois, um de 0 a $r_{i}$ (para $r_{j} \leq r_{i}$ ) e o outro de $r_{i}$ a $\infty$ (para $r_{j} \geq r_{i}$ ), o que leva ao seguinte resultado final:

$$
k e\left[\int_{0}^{r_{i}} \frac{\left[P_{n_{j} \ell_{j}}\left(r_{j}\right)\right]^{2}}{r_{i}} d r_{j}+\int_{r_{i}}^{\infty} \frac{\left[P_{n_{j} \ell_{j}}\left(r_{j}\right)\right]^{2}}{r_{j}} d r_{j}\right]
$$

\section{REFERÊNCIAS}

1. Hartree, D. R.; Proc. Cambridge Phil. Soc. 1928, 24, 89.

2. Hartree, D. R.; Proc. Cambridge Phil. Soc. 1928, 24, 111.

3. Levine, I. N.: Quantum Chemistry, $6^{\text {th }}$ ed., Pearson Education: New Jersey, 2009.

4. Eyring, H.; Walter, J.; Kimball, G. E.; Quantum Chemistry, John Wiley \& Sons: New York, 1944.

5. Pilar, F. L.; Elementary Quantum Chemistry, $2^{\text {nd }}$ ed., Dover: New York, 2001.

6. Bartell, L. S.; Brockway, L. O.; Physical Review 1953, 90, 833.

7. Messiah, A.; Quantum Mechanics, North Holland Publ. Co.: Amsterdam and John Wiley \& Sons: New York, 1966, vol. I.

8. Tipler, P. A.; Mosca, G.; Physics for Scientists and Engineers (Extended Version), $5^{\text {th }}$ ed., W. H. Freeman \& Company: New York \& Basingstoke, 2003.

9. Slater, J. C.; Quantum Theory of Atomic Structure, $1^{\text {st }}$ ed., McGraw-Hill: New York, 1960, vol. 1.

10. Hartree, D. R.; The Calculation of Atomic Structures, John Wiley \& Sons, Inc: New York, 1957.

11. Szabo, A.; Ostlund, N. S.; Modern Quantum Chemistry, $1^{\text {st }}$ ed. revised, Dover: New York, 1996.

12. Duarte, H. A.; Química Nova na Escola 2003, n 17, 22.

13. Peixoto, E. A.; Teoria Quântica, Ed. USP: São Paulo, 1988.

14. Berry, R. S.; Rice, S. A.; Ross, J.; Physical Chemistry, John Wiley \& Sons, Inc: New York, 1980.

15. Hartree, D. R.; Proc. R. Soc. Lond. A 1933, 141, 282.

16. Slater, J. C.; Phys. Rev., 1930, 35, 210.

17. Fock, V. A.; Z. Physik 1930, 61, 126.

18. Unsöld, A.; Ann. Physik 1927, 82, 355.

19. Hartree, D. R.; Black, M. M.; Proc. R. Soc. Lond. A 1933, 139, 311

20. Hartree, D. R.; Proc. Cambridge Phil. Soc. 1928, 24, 426.

21. Slater, J. C.; Phys. Rev. 1929, 34, 288

22. Fock, V. A.; Z. Physik 1930, 62, 795.

23. Fock, V. A.; Petrashen, M.; Physik. Z. Sowjetunion 1934, 6, 368. 